\title{
HARMONICS ON STIEFEL MANIFOLDS AND GENERALIZED HANKEL TRANSFORMS
}

\author{
BY STEPHEN S. GELBART
}

Communicated by W. Fuchs, November 4, 1971

Introduction and notation. The following two theorems are basic to the classical theory of spherical harmonics and their importance in analysis is well known.

TheOREM 1. (CARTAN [2].) Suppose $p$ is a function on the $(n-1)$-sphere $S^{n-1}$ which transforms under $S O(n)$ according to an irreducible representation of highest weight $(k, 0, \ldots, 0)$. Then $p$ extends to a harmonic polynomial on $\boldsymbol{R}^{n}$ satisfying the homogeneity condition $p(r X)=r^{k} p(X)$ for all $r>0$ and $X \in \boldsymbol{R}^{n}$.

THEOREM 2. (BOCHNER [1].) Suppose $f$ is a radial function on $\boldsymbol{R}^{n}, p$ is as in Theorem 1 , and $F=f p$ is square-integrable. Then the Fourier transform of $F$ is $g p$ where $g$ is the Hankel transform of $f$ of order $k+((n-2) / 2)$.

In this note we announce an extension of these theorems to the setting of Stiefel manifolds and matrix space. Our work makes it possible to construct holomorphic discrete series representations for the real symplectic group by decomposing a tensor product of certain projective representations introduced earlier by Shale and Weil. (See Weil [11] and also Shalika [10].) Proofs of the results announced here and their application to the construction of discrete series will appear elsewhere.

We let $M_{n, m}$ denote the $n \times m$ real matrix space, $S^{n, m}$ the Stiefel manifold of matrices $V \in M_{n, m}$ such that ${ }^{t} V V=I_{m}$, and $P_{m}$ the cone of $m \times m$ positive-definite symmetric matrices. The rotation group $S O(n)$ acts on $S^{n, m}$ and $M_{n, m}$ by left matrix multiplication so that $S^{n, m} \cong S O(n) / S O(n-m)$. Corresponding to the decomposition $M_{n, m}=S^{n, m} \times P_{m}$ we have the integral formula

$$
\int_{M_{n, m}} F(X) d X=c_{n, m} \int_{P_{m}}\left(\int_{S^{n, m}} F\left(V R^{1 / 2}\right) d V\right)(\operatorname{det} R)^{v} d R
$$

where $v=(n-m-1) / 2, c_{n, m}$ is a constant depending only on $n$ and $m$, and $d V$ is $S O(n)$-invariant. The algebra $M_{m, m}$ acts on $M_{n, m}$ by right matrix multiplication.

AMS 1969 subject classifications. Primary 2257, 4216; Secondary 2265, 3327.

Key words and phrases. Generalized spherical harmonics, Stiefel manifold, matrix space, class $m$ representations of $S O(n)$, holomorphic representations of the general linear group, Fourier transforms on matrix space, generalized Hankel transforms, Bessel functions of matrix argument. 
Let $L$ denote the restriction of the left regular representation of $S O(n)$ to $L^{2}\left(S^{n, m}\right)$. We shall refer to irreducible representations of $S O(n)$ which occur in $L$ as class $m$ representations and write

$$
L^{2}\left(S^{n, m}\right)=\sum_{\omega} \bigoplus H_{\omega}^{n, m}
$$

where $H_{\omega}^{n, m}$ consists of functions which transform according to the (class $m$ ) representation $\omega$. We call the functions in $H_{\omega}^{n, m}$ Stiefel harmonics of order $\omega$.

Statement of results.1. Throughout this section we assume $n>2 m$.

Lemma 1. Let $\left(m_{1}, \ldots, m_{[n / 2]}\right)$ denote the highest weight vector of an irreducible representation of $S O(n)$. Then the representation is class $m$ if and only if $m_{j}=0$ for $j>m$.

We fix, once and for all, an $m$-tuple of nonnegative integers $\omega$ $=\left(m_{1}, \ldots, m_{m}\right)$ with $m_{1} \geqq m_{2} \geqq \cdots \geqq m_{m}$. We let $\rho_{\omega}$ denote the uniquely determined holomorphic irreducible representation of $G L(m, C)$ whose restriction to $U(m)$ has highest weight $\omega$ and let $\omega$ itself denote the class $m$ representation of $S O(n)$ of highest weight $\left(m_{1}, m_{2}, \ldots, m_{m}, 0, \ldots, 0\right)$.

LEMMA 2. The class representation $\omega$ occurs in $L^{2}\left(S^{n, m}\right)$ with multiplicity equal to the dimension of $\rho_{\omega}$. (Caution: the dimension of $\rho_{\omega}$ should not be confused with the dimension of $\omega$; this is not ordinary Frobenius reciprocity.)

Lemma 2 suggests an intimate relation between the class $m$ representations of $S O(n)$ and the so-called integral holomorphic representations of $G L(m, C)$. This relation is made explicit by the results below and is established via the Frobenius-Schur-Young-Weyl theory of symmetry classes in tensor space. We let $r_{\omega}$ denote the dimension of $\rho_{\omega}$ (hence also the multiplicity of $\omega$ in $\left.L^{2}\left(S^{n, m}\right)\right)$. If $\rho_{\omega}(A)$ is some matrix realization of $\rho_{\omega}$ we call a $C^{r \omega}$-valued function on $M_{n, m}$ homogeneous of degree $\rho_{\omega}(A)$ if the function transforms under $M_{m, m}$ according to $\rho_{\omega}(A)$, i.e.,

$$
p(X A)=p(X) \rho_{\omega}(A)
$$

for all $A \in M_{m, m} .\left(\rho_{\omega}(A)\right.$ extends from $G L(m, C)$ to all $m \times m$ complex matrices, invertible or not, since $m_{m} \geqq 0$.) Following Helgason [4] we call a function on $M_{n, n} S O(n)$-harmonic if it is annihilated by all $S O(n)$-invariant constant coefficient differential operators without constant term (generalized "Laplacians").

PRoposition 1. There exists a matrix representation $\rho_{\omega}(A)$ and a space $P^{o s}$

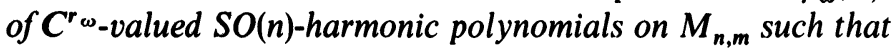

(1) each $p \in P^{\omega}$ is homogeneous of degree $\rho_{\omega}(A)$,

(2) as an $S O(n)$-module, $P^{\omega}$ is equivalent to $\omega$. 
Lemma 2 is crucial to the proof of Proposition 1. The basic idea is to construct $\rho_{\omega}(A)$ directly via tensor theory and then define a special element of $P^{\omega}$ (a cyclic vector for $\omega$ ) in terms of $\rho_{\omega}(A)$. If $m=1$ and $\omega=k$ then $P^{\omega}$ is the classical space of solid spherical harmonics of order $k$ on $\boldsymbol{R}^{\boldsymbol{m}}$. More generally we define the space $\mathscr{H}_{\omega}^{n, m}$ of solid harmonics of order $\omega$ on $M_{n, m}$ to be the direct sum of the spaces $\mathscr{H}_{\omega}^{j}$ of $j$ th coordinate functions of elements of $P^{\omega}$.

THEOREM 3. A Stiefel harmonic of order $\omega$ on $S^{n, m}$ extends to a solid harmonic of the same order on $M_{n, m}$.

To obtain an analogue of Theorem 2 we need first to strengthen Theorem 3.

Theorem 3'. Let $\rho_{\omega}(A)$ be as in Proposition 1. Then there is a matrix realization $\omega(0)$ of the representation $\omega$ of $S O(n)$ satisfying the following property: if $\omega^{*}(0)$ denotes the upper left hand $r_{\omega} \times r_{\omega}$ submatrix of $\omega(0)$ then $\omega^{*}\left(0^{-1}\right)^{t}$ is well-defined as a function of $S^{n, m}$ and extends to a $C^{{ }^{r} \omega \times r_{\omega}}$ valued $S O(n)$-harmonic polynomial on $M_{n, m}$ which is homogeneous of degree $\rho_{\omega}(A)$.

2. Let ${ }^{*} \mathscr{H}_{\omega}^{n, m}$ denote the subspace of $L^{2}\left(M_{n, m}\right)$ which transforms under $S O(n)$ according to the representation $\omega$. Then

$$
L^{2}\left(M_{n, m}\right)=\sum_{\omega} \bigoplus * \mathscr{H}_{\omega}^{n, m}
$$

and functions in $* \mathscr{H}_{\omega}^{n, m}$ are of the form

$$
F\left(V R^{1 / 2}\right)=\sum_{i=1}^{r_{\omega}} \sum_{j=1}^{d_{\omega}} f_{i j}(R) t_{i j}^{\omega}\left(0^{-1}\right)
$$

where $f_{i j}$ belongs to $L^{2}\left(P_{m}, c_{n, m}(\operatorname{det} R)^{v} d R\right)$ and $\left(t_{i j}^{\omega}(0)\right)$ is the matrix $\omega(0)$ described in Theorem $3^{\prime}$. (Decompositions of this type are discussed in greater generality by Gross and Kunze in [3].) Thus ${ }^{*} \mathscr{H}_{\omega}^{n, m}$ is nonempty if and only if $\omega$ is class $m$.

We recall the Fourier operator on $M_{n, m}$ as an isometry of $L^{2}\left(M_{n, m}\right)$ which commutes with the action of $S O(n)$ and hence preserves the decomposition (3).

Lemma 3. Suppose $F$ in $* \mathscr{H}_{\omega}^{n, m}$ corresponds to the matrix $\left(f_{i j}\right)$ (as in (4)) and let $S_{\omega}^{n, m}(R, P)$ on $P_{m} \times P_{m}$ be defined by

$$
S_{\omega}^{n, m}(R, P)=c_{n, m} \int_{S O(n)} \omega^{*}\left(0^{-1}\right)^{t} \exp \left[2 \pi i \operatorname{trace}\left({ }^{(} \operatorname{T} 0 T R^{1 / 2} P^{1 / 2}\right)\right] d 0 .
$$

Then the Fourier transform of $F$ again belongs to ${ }^{*} \mathscr{H}_{\omega}^{n, m}$ and corresponds to 
the matrix $\left(g_{i j}\right)$ where

$$
\left(g_{i j}(P)\right)=\int S_{\omega}^{n, m}(R, P)\left(f_{i j}(R)\right)(\operatorname{det} R)^{v} d R .
$$

We define the Hankel transform of order $\omega$ on $P_{m}$ to be the restriction of the Fourier operator to ${ }^{*} \mathscr{H}_{\omega}^{n, m}$ and we denote it by $U_{\omega}^{n, m}$; the matrix-valued kernel $S_{\omega}^{n, m}(R, P)$ is the Bessel kernel of order $\omega$ on $P_{m}$. By combining Theorem $3^{\prime}$ with a Laplace transform argument we obtain an explicit formula for $S_{\omega}^{n, m}(R, P)$ (and hence $U_{\omega}^{n, m}$ ).

Theorem 4. Let $\rho_{\omega}(A)$ be as in Proposition 1 and let $Z=A+i B$ denote a complex symmetric $m \times m$ matrix. Then

$$
\begin{aligned}
& S_{\omega}^{n, m}(R, P)(\operatorname{det} R)^{v} \\
& =\left\{\frac{\pi^{m n / 2} \rho_{\omega}\left(i P^{1 / 2}\right)}{(2 \pi i)^{m(m+1) / 2}} \int_{Z=A_{0}+i B ; A_{0}>0}(\operatorname{det} Z)^{-n / 2} \rho_{\omega}\left(\pi^{-1} Z\right)^{-1}\right. \\
& \left.\exp \left[\operatorname{trace}(R Z)-\operatorname{trace}\left(\pi^{2} P Z^{-1}\right)\right] d z\right\} \rho_{\omega}\left(R^{1 / 2}\right)^{-1} .
\end{aligned}
$$

Theorem 4 leads to a generalization of the classical "Bochner relations" for Fourier transforms. (See, for example, [8, p. 72].)

THEOREM 5. If $\omega=\left(m_{1}, \ldots, m_{m}\right)$ let $\omega^{\prime}$ denote $\left(m_{1}-m_{m}, m_{2}-m_{m}, \ldots, 0\right)$ and set $n^{\prime}$ equal to $n+2 m_{m}$. Then $U_{\omega}^{n, m}=i^{m m_{m}} U_{\omega^{\prime}}^{n^{\prime}, m}$. (Equality here means that $U_{\omega}^{n, m}$ and $U_{\omega}^{n^{\prime}, m}$ coincide as operators on the isomorphic spaces $*^{*} \mathscr{H}_{\omega}^{n, m}$ and $* \mathscr{H}_{\omega^{\prime}}^{n^{\prime}, m}$.)

SOME REMARKS. Examples of generalized spherical harmonics have appeared before in the literature. In [5] Herz introduced a class of harmonic polynomials on $M_{n, m}$ satisfying the homogeneity condition $p(X A)$ $=(\operatorname{det} A)^{k} p(X)$ for all $A \in M_{m, m}$. In our context these polynomials are solid harmonics of order $\omega=(k, k, \ldots, k)$ and hence correspond to representations of $S O(n)$ which occur only once in $L^{2}\left(S^{n, m}\right)$. Generalized spherical harmonics also appear in Levine [6] (for the case $m=2$ ), and Helgason [4]. It is clear from [6] that our solid harmonics span the space of all $S O(n)$ harmonic functions on $M_{n, m}$ when $m=2$. It is not clear what the relation is between our harmonics and those introduced by Maas in [7].

It would also be of interest to find an intrinsic characterization of general order solid harmonics on $M_{n, m}$ and to obtain analogues of the results announced here for $n<2 m$ (in particular, for $n=m$; cf. [9, Problem 7]). (The assumption that $n$ be strictly greater than $2 m$ is not necessary but was made in order to avoid certain technicalities related to the representation theory of $S O(n)$.) 
ACKNOWLEDGements. It is a pleasure to thank E. M. Stein for the many crucial suggestions and criticisms he contributed to this work.

\section{REFERENCES}

1. S. Bochner, Theta relations with spherical harmonics, Proc. Nat. Acad. Sci. U.S.A. 37 (1951), 804-808. MR 14, 43.

2. E. Cartan, Sur la determination d'un système orthogonal complet dans un espace de Riemann symétrique clos, Rend. Circ. Mat. Palermo 53 (1929), 217-252.

3. K. Gross and R. Kunze, Fourier decompositions of certain representations, Geometry and Analysis of Symmetric Spaces, Marcel Dekker, New York, 1972.

4. S. Helgason, Invariants and fundamental functions, Acta Math. 109 (1963), 241-258. MR 29 \# 3581.

5. C. S. Herz, Bessel functions of matrix argument, Ann. of Math. (2) 61 (1955), 474-523. MR 16, 1107.

6. D. Levine, Systems of singular integrals on spheres, Trans. Amer. Math. Soc. 144(1969), 493-522.

7. H. Maas, Spherical functions and quadratic forms, J. Indian Math. Soc. 20 (1956), 117-162. MR 19, 252.

8. E. Stein, Singular integrals and differentiability properties of functions, Princeton Univ. Press, Princeton, N.J., 1970.

9. - Some problems in harmonic analysis suggested by semisimple Lie groups and symmetric spaces, Internat. Congress of Math., Nice, 1970.

10. J. Shalika, Representations of the two-by-two unimodular group over local fields, Seminar on Representations of Lie Groups, Princeton University, Princeton, N.J., 1966.

11. A. Weil, Sur certains groupes d'opérateurs unitaires, Acta Math. 111 (1964), 143-211. MR 29 \# 2324.

Department of Mathematics, Cornell University, Ithaca, New York 14850 